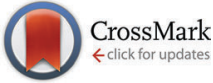

Cite this: Phys. Chem. Chem. Phys., 2016, 18, 11132

Received 15th December 2015, Accepted 30th March 2016

DOI: $10.1039 / c 5 c p 07752 d$

www.rsc.org/pccp

\section{Photo-degradation in air of the active layer components in a thiophene-quinoxaline copolymer:fullerene solar cell $\dagger$}

\author{
Rickard Hansson, ${ }^{a}$ Camilla Lindqvist, ${ }^{a}$ Leif K. E. Ericsson, ${ }^{a}$ Andreas Opitz, ${ }^{b}$ \\ Ergang Wang ${ }^{c}$ and Ellen Moons ${ }^{{ }^{a}}$
}

\begin{abstract}
We have studied the photo-degradation in air of a blend of $[6,6]$-phenyl- $C_{61}$-butyric acid methyl ester (PCBM) and poly[2,3-bis-(3-octyloxyphenyl)quinoxaline-5,8-diyl-alt-thiophene-2,5-diyl] (TQ1), and how the photo-degradation affects the solar cell performance. Using near-edge $X$-ray absorption fine structure (NEXAFS) spectroscopy, changes to the electronic structure of TQ1 and PCBM caused by illumination in ambient air are investigated and compared between the pristine materials and the blend. The NEXAFS spectra show that the unoccupied molecular orbitals of TQ1 are not significantly changed by the exposure of pristine TQ1 to light in air, whereas those of PCBM are severely affected as a result of photo-induced degradation of PCBM. Furthermore, the photo-degradation of PCBM is accelerated by blending it with TQ1. While the NEXAFS spectrum of TQ1 remains unchanged upon illumination in air, its valence band spectrum shows that the occupied molecular orbitals are weakly affected. Yet, UV-Vis absorption spectra demonstrate photo-bleaching of TQ1, which is attenuated in the presence of PCBM in blend films. Illumination of the active layer of TQ1:PCBM solar cells prior to cathode deposition causes severe losses in electrical performance.
\end{abstract}

\section{Introduction}

Polymer solar cells are a promising alternative to conventional solar cell technologies due to their compatibility with printing techniques, mechanical flexibility and the possibility to produce light-weight modules. Power conversion efficiencies (PCE) have been steadily increasing over the last decades and single junction devices are now showing a PCE above $10 \% .^{1,2}$ This makes stability one of the most important current challenges in the field of polymer solar cell research. ${ }^{3,4}$

The active layer of a polymer solar cell typically consists of a mixture of an electron donating conjugated polymer and an electron accepting fullerene derivative. One of the most commonly used fullerene derivatives is [6,6]-phenyl- $\mathrm{C}_{61}$-butyric acid methyl ester (PCBM, Fig. 1).

The degradation of the electrical performance of polymer solar cells is a complex phenomenon that involves several chemical and physical processes in different parts of the device. The donor and acceptor components, the active layer morphology,

\footnotetext{
${ }^{a}$ Department of Engineering and Physics, Karlstad University, 65188 Karlstad, Sweden. E-mail: ellen.moons@kau.se

${ }^{b}$ Department of Physics, Humboldt-Universität zu Berlin, 12489 Berlin, Germany

${ }^{c}$ Department of Chemistry and Chemical Engineering, Chalmers University of Technology, 41296 Göteborg, Sweden

$\dagger$ Electronic supplementary information (ESI) available. See DOI: 10.1039/c5cp07752d
}
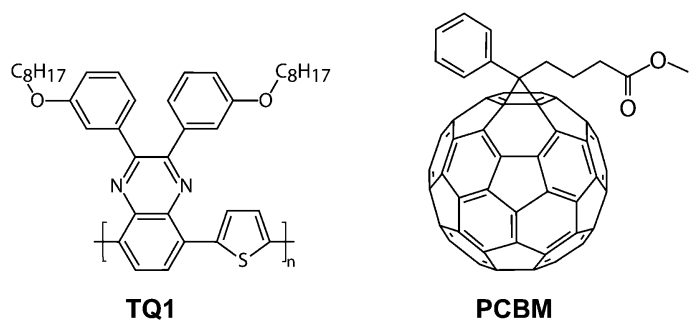

Fig. 1 Molecular structures of TQ1 and PCBM.

as well as the interlayers and electrode materials have all been shown to take part in device degradation. ${ }^{4-8}$ The electrical degradation of polymer solar cells is often assigned to ingress of oxygen and/or water into the device. Oxygen can both dope the photoactive layer components and assist in photochemical oxidation reactions that cause a decrease in performance. ${ }^{9}$

The photo-degradation of the light-absorbing electron donor material has been investigated for a number of well-known polymers. ${ }^{10-15}$ In most polymer/fullerene blends reported so far the polymer photo-oxidizes when exposed to light in ambient conditions. ${ }^{16,17}$ Reese $e t$ al. showed that a film of the common donor polymer poly-hexylthiophene (P3HT) photo-bleaches, while accompanied by PCBM in a blend, the photo-bleaching is inhibited. ${ }^{17}$ Also X-ray absorption spectroscopy shows that blending with 
PCBM slows down the degradation of $\mathrm{P} 3 \mathrm{HT}$ by a factor of $3 .{ }^{18} \mathrm{In}$ a recent study by Tournebize et al., however, the stabilization of P3HT by PCBM at short illumination times $(<10 \mathrm{~h})$ is interpreted as due to morphological reorganization of the components in the active layer, while photochemical degradation was dominant over reorganization at longer illuminations times. ${ }^{19}$ It is however unlikely that such morphological reorganization is dominant in polymer systems with higher glass transition temperature. Based on the studied degradation mechanisms, several polymers have been suggested that have a better resistance towards photochemical degradation, ${ }^{13,14}$ and some rules of thumb for photochemical stability of polymers were formulated by Manceau et $a l .{ }^{11}$ As increasingly stable polymers are being developed, the importance of studying the other component of the active layer, i.e. the fullerene derivative, becomes more and more apparent.

Poly[2,3-bis-(3-octyloxyphenyl)quinoxaline-5,8-diyl-alt-thiophene2,5-diyl] (TQ1, Fig. 1) is a polymer ${ }^{20}$ that has demonstrated a high PCE of $7 \%{ }^{21}$ and a substantially improved chemical stability compared to P3HT. ${ }^{14}$ This makes TQ1 suitable for the present study where we want to distinguish the degradation of the fullerene derivative from that of the polymer.

The stability of PCBM has previously been studied by Reese et al. who observed oxidation of PCBM upon exposure of a P3HT: PCBM blend film to white light in ambient air for 1000 hours. ${ }^{17}$ By using Fourier transform infrared spectroscopy, Chambon et al. showed that the photo-oxidation of PCBM mainly involves the oxidation of the $\mathrm{C}_{60}$ moiety. ${ }^{22}$ Yamane et al. have studied the photo-oxidation of PCBM in blends with P3HT, using matrixassisted laser desorption/ionization time-of-flight (MALDI-TOF) mass spectrometry, their findings suggesting that PCBM is stabilized by $\mathrm{P} 3 \mathrm{HT} .{ }^{23}$ Recently, the effect of light exposure in air on the electronic structure of PCBM was studied by Anselmo et al. using a combination of near-edge X-ray absorption fine structure (NEXAFS) spectroscopy and X-ray photoelectron spectroscopy. ${ }^{24}$ Considerable changes to both the occupied and unoccupied molecular states were found, meaning that the surface of a PCBM thin film is significantly affected by exposure to light in ambient air. The question remains how this surface degradation of pristine PCBM compares to that of PCBM in a blend, as the one used in a solar cell device, as well as how the photo-degradation of the active layer components affects the solar cell performance.

Here we report on the photo-induced changes to the electronic structure of PCBM and TQ1, in their pristine forms as well as in a blend, and we evaluate the effect of the degradation on the solar cell performance.

\section{Experimental section}

\subsection{Materials}

The synthesis of TQ1 is described elsewhere. ${ }^{20}$ The number average $\left(M_{\mathrm{n}}\right)$ and weight average $\left(M_{\mathrm{w}}\right)$ molecular weights, determined by size exclusion chromatography relative to polystyrene standards, were $M_{\mathrm{n}}=34 \mathrm{~kg} \mathrm{~mol}{ }^{-1}$ and $M_{\mathrm{w}}=91 \mathrm{~kg} \mathrm{~mol}{ }^{-1}$. The eluent was 1,2,4-trichlorobenzene and the working temperature $135{ }^{\circ} \mathrm{C}$. The mass density of TQ1 is estimated to be $1 \mathrm{~g} \mathrm{~cm}^{-3}$.
PCBM (purity $>99.5 \%$ ) was purchased from Solenne BV (The Netherlands). Reported mass density values for PCBM range from $1.3-1.6 \mathrm{~g} \mathrm{~cm}^{-3} \cdot{ }^{25-27}$ Here the value of $1.5 \mathrm{~g} \mathrm{~cm}^{-3}$ is used.

Poly(3,4-ethylenedioxythiophene) polystyrene sulfonate (PEDOT: PSS) (Clevios P VP AI 4083) was purchased from Heraeus Precious Metals $\mathrm{GmbH} \& \mathrm{Co}$. KG.

Chlorobenzene (analytical grade) was purchased from Merck $\mathrm{KGaA}$ and was used as received.

For the NEXAFS and valence band characterizations, substrates were cut from silicon wafers (n-type, (001) orientation and resistivity $0.001-0.003 \Omega \mathrm{cm}$ ) and cleaned using the standard RCA-method, ${ }^{28-30}$ without the final HF-etching step in order to leave the surface hydrophilic. For UV-Vis characterization, glass substrates, also cleaned using the RCA-method, were used.

For solar cells, patterned ITO-coated glass substrates from Kintec Company, China, with a $200 \mathrm{~nm}$ thick ITO layer with sheet resistivity $10 \Omega \mathrm{sq}^{-1}$ were used, which were cleaned in isopropanol in an ultrasonic bath for $60 \mathrm{~min}$ and subsequently UV-ozone treated for 20 minutes.

\subsection{Sample preparation}

Blend solutions of TQ1 : PCBM were prepared in chlorobenzene in a $1: 3$ weight/weight ratio, except for the UV-Vis blend sample where a $10: 1$ ratio was also used.

The highest efficiencies for TQ1-based solar cells were reported for $[6,6]$-phenyl $\mathrm{C}_{71}$-butyric acid methyl ester $\left(\mathrm{PC}_{70} \mathrm{BM}\right)$ as the electron acceptor and for solution formulations involving high vapour pressure solvents and additives. ${ }^{21}$ Apart from giving high efficiencies, such solution formulations also give films with high TQ1 surface concentrations. ${ }^{31}$ Since our objective here is to investigate how the PCBM at the surface of the active layer is affected by the exposure to air and light, we do, rather than aiming at the highest possible efficiencies, choose a solution formulation that will lead to a non-negligible surface concentration of PCBM in the blend film that can be detected by its NEXAFS resonances. We also chose PCBM rather than $\mathrm{PC}_{70} \mathrm{BM}$ as the acceptor material since the NEXAFS $\pi^{*}$ resonances of PCBM overlap less with those of TQ1 than is the case for $\mathrm{PC}_{70} \mathrm{BM}$. Note that this choice will lead to lower device performance compared to $\mathrm{PC}_{70} \mathrm{BM}^{20}$

The active layer was deposited by spincoating in a protected $\mathrm{N}_{2}$ atmosphere $\left(<0.1\right.$ ppm $\mathrm{O}_{2},<0.1$ ppm $\left.\mathrm{H}_{2} \mathrm{O}\right)$ inside a glove box (M. Braun Inertgas-Systeme $\mathrm{GmbH}$ ) at $750 \mathrm{rpm}$ for $100 \mathrm{~s}$. In addition to the blend films, films of pristine TQ1 and pristine PCBM (solutions in chlorobenzene) were also prepared by spincoating.

For NEXAFS spectroscopy, valence band spectroscopy and solar cells, a $50 \mathrm{~nm}$ PEDOT:PSS layer was spincoated on the substrates after filtering it through a $0.45 \mu \mathrm{m}$ nylon filter. The PEDOT:PSS covered substrates were annealed at $120{ }^{\circ} \mathrm{C}$ for 20 minutes in a vacuum oven under low vacuum to remove any remaining water.

The film thicknesses were measured by scanning across a scratch in the film with the tip of an AFM (Nanoscope IIIa Multimode, Veeco Metrology group) in tapping mode, using a Si tip. The film thickness of pristine TQ1, pristine PCBM and all blends was $90 \pm 5 \mathrm{~nm}$. 
Light exposure was performed in air using AM1.5 illumination from a solar simulator (Sol2A, model 94022A, Oriel Instruments, USA).

For the solar cell fabrication, the top electrodes were deposited by subsequent thermal evaporation of $0.3 \mathrm{~nm} \mathrm{LiF}$ and $100 \mathrm{~nm} \mathrm{Al}$ on of the unexposed active layers as well as on the light-exposed active layers. (Univex $350 \mathrm{G}$, Oerlikon Leybold Vacuum $\mathrm{GmbH}$ ). The deposition rates were $0.5 \AA_{\mathrm{s}^{-1}}$ for $\mathrm{LiF}$ and $1 \mathrm{\AA} \mathrm{s}^{-1}$ for $\mathrm{Al}$ and the pressure was in the range of $10^{-6}$ mbar during the evaporation.

\subsection{Characterization}

NEXAFS spectroscopy and X-ray photoelectron spectroscopy were carried out at beamline D1011 of the MAX-IV National Laboratory for Synchrotron Radiation in Lund, Sweden. All NEXAFS spectra were collected near the C1s absorption edge in the photon energy range $276-327 \mathrm{eV}$ at $55^{\circ}$ incident angle with respect to the sample surface. Total electron yield (TEY) spectra were obtained by measuring the sample drain current. Reference spectra were recorded on a gold coated mica sample (Georg Albert, PVD-Beschichtungen) that had been cleaned in situ by sputtering with argon. All NEXAFS spectra were divided by the gold spectrum and normalized in the high photon energy region. $^{32,33}$ The photon energy scale was calibrated by measuring the spectrum of highly ordered pyrolytic graphite (HOPG) and using the position of the exciton resonance at $291.65 \mathrm{eV}$ as an energy reference. ${ }^{34}$ The C1s NEXAFS spectrum from a metal grid was measured simultaneously to all the samples and used to compensate for any drift in photon energy from the monochromator.

The surface composition of the blend films was obtained from the NEXAFS spectra by finding the linear combination of the pure components' spectra that best matched the spectrum of the blend. The coefficients in that linear combination then give the volume ratio of the components. ${ }^{35}$ This assumes that the pure components do not react in the blend forming new components and that the electron scattering length is similar in both components. No degradation of the samples due to exposure to the X-ray beam was observed during the NEXAFS measurements.

X-ray photoelectron spectroscopy was used to collect the valence band spectra of TQ1 films using a photon energy of $150 \mathrm{eV}$ and a SCIENTA SES200 electron-energy analyzer. Spectra were collected in normal emission and were energy calibrated with respect to the Fermi level measured on a gold surface that had been cleaned in situ by argon sputtering.

UV-Vis absorption measurements on the TQ1 and blend films were performed in transmission mode using a Cary 5000 UV-Vis-NIR spectrophotometer (Agilent Technologies).

The current-voltage characteristics of the solar cells were measured in the dark as well as under illumination (AM1.5) using a Keithley 2636A Dual-channel SourceMeter, in a sealed cell. The cell is filled with $\mathrm{N}_{2}$ slightly above atmospheric pressure and has a window made of a $2 \mathrm{~mm}$ thick poly(methyl methacrylate) (PMMA) sheet. No spectral correction was made for any UV light absorption by the PMMA. When extracting the photovoltaic parameters, the data points were interpolated using a cubic spline. The series resistance was obtained by fitting the higher voltage part of the forward dark curve to the Shockley equation:

$$
j=j_{0}\left[\exp \left(\frac{e\left(V-j R_{\mathrm{s}}\right)}{n k_{\mathrm{B}} T}-1\right)\right]
$$

where $j$ is the current density, $j_{0}$ the reverse bias saturation current density, $e$ the electron charge, $V$ the applied voltage, $R_{\mathrm{S}}$ the series resistance, $n$ the ideality factor, $k_{\mathrm{B}}$ Boltzmann's constant, and $T$ the absolute temperature, with $j_{0}, R_{\mathrm{S}}$ and $n$ being the fitting parameters.

\section{Results}

C1s NEXAFS spectra of thin films of pristine TQ1, pristine PCBM and a TQ1:PCBM 1:3 w/w blend, before and after exposure to AM1.5 light in air for different times are shown in Fig. 2. As can be seen in Fig. 2a, the TQ1 spectrum is not significantly affected by exposure to light in air. The PCBM spectrum, on the other hand, is strongly affected by this exposure. The intensity of the $\pi^{*}$ resonances at $284.5 \mathrm{eV}$, $285.8 \mathrm{eV}$ and $286.2 \mathrm{eV}$ are reduced, while the peak at $288.4 \mathrm{eV}$ grows with increasing exposure time, in agreement with what has been reported by Anselmo et al. ${ }^{24}$ By density functional theory, the peak at $284.5 \mathrm{eV}$ has earlier been assigned to the $\mathrm{C}_{60}$ fullerene and phenyl moieties of PCBM, while the peaks at $285.8 \mathrm{eV}$ and $286.2 \mathrm{eV}$ mainly arise from the $\mathrm{C}_{60}$ cage. ${ }^{36}$ The peak at $288.4 \mathrm{eV}$ arises from transitions to $\sigma^{*}$ molecular orbitals, ${ }^{37}$ and it contains contributions from the side chain as well as from the fullerene cage. ${ }^{36}$ The observed changes in the PCBM NEXAFS spectra upon light exposure in air imply double bonds being broken and single bonds being formed, i.e. a transition from $\mathrm{sp}^{2}$ to $\mathrm{sp}^{3}$ hybridized carbon. The NEXAFS spectrum of the TQ1:PCBM blend, shown in Fig. 2c, consists of contributions from PCBM and TQ1, and is also modified by the light exposure in air; in particular in the $\pi^{*}$ region where the resonances corresponding to PCBM decrease in intensity with increased exposure time.

The valence band spectrum of TQ1, before and after exposure to light in air, is shown in Fig. 3a. The onset of the valence band is not affected by the exposure. With increasing exposure time, the intensity of the peak at binding energy $3 \mathrm{eV}$ is reduced. This peak can be assigned to the highest occupied molecular orbital (HOMO) of the polymer.

Fig. 3b shows UV-Vis absorption spectra of pristine TQ1 after different exposure times to light in air. The absorption peak intensities decrease with increasing exposure time due to photobleaching of the polymer, and there are no significant energy shifts. Also, the low and high energy peaks decrease at slightly different rates. Fig. $3 c$ shows the remaining absorbance calculated from the low energy absorption peak of pristine TQ1 films and TQ1 with $10 \mathrm{wt} \%$ PCBM for the different exposure times. For longer exposure times it can be seen that adding $10 \mathrm{wt} \%$ PCBM slows down the photo-bleaching of TQ1, in agreement with what has previously been reported by Tromholt et $a .^{38}$

Fitting the unexposed TQ1:PCBM 1:3 w/w blend NEXAFS spectrum in Fig. $2 \mathrm{c}$ to a linear combination of the pristine 


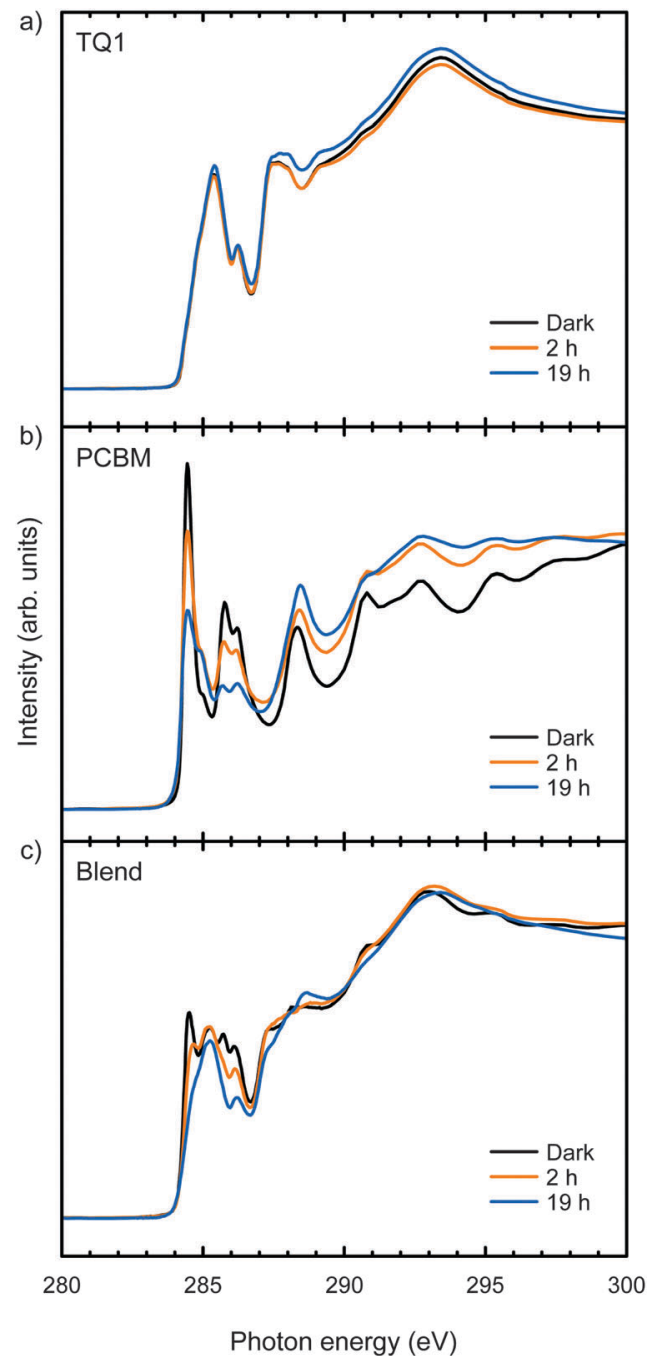

Fig. 2 C1s NEXAFS spectra (TEY) of spincoated films of (a) pristine TQ1, (b) pristine PCBM and (c) a TQ1: PCBM 1:3 w/w blend unexposed to air and light (black), and exposed to air and AM1.5 light for different times (orange and blue).

components' spectra gives a surface composition of $32 \%$ (vol) PCBM (fit shown in the ESI $\dagger$ ). This result is in agreement with what was shown in previous studies, i.e. that the surface of TQ1:PC ${ }_{70} \mathrm{BM}$ films is TQ1-enriched. ${ }^{31}$ The PCBM component in the NEXAFS spectrum of a TQ1:PCBM blend films that has been exposed to light in air can be extracted from the blend spectrum by subtracting the TQ1 component from the unexposed blend spectrum, using the fact that the NEXAFS spectrum of pristine TQ1 is unaffected by the exposure (Fig. 2a). This is also justified by the UV-Vis measurements that show that PCBM protects TQ1 from photo-bleaching (Fig. 3). Since the NEXAFS spectrum of pristine TQ1 is not significantly affected by light exposure in air, we can assume that the TQ1 component in a blend with PCBM is likely to be affected even less.

Fig. 4a shows C1s NEXAFS spectra of the PCBM component in TQ1:PCBM blends, unexposed as well as exposed to light in air for different times. These spectra were obtained by subtracting the TQ1 component from the NEXAFS spectra of the blends, shown in Fig. 2c. Comparing the effect of the exposure on the NEXAFS spectrum of PCBM in a blend film (Fig. 4a) to that of pristine PCBM (Fig. 2b), it can be seen that the decrease in relative peak intensity resulting from the exposure is larger for PCBM in a blend with TQ1 than for pristine PCBM. The relative peak area of the $\pi^{*}$ resonance at $284.5 \mathrm{eV}$ as a function of exposure time is shown in Fig. $4 \mathrm{~b}$ for pristine PCBM and the PCBM component in the blend. It is clear that the PCBM component in the blend is affected much stronger by the exposure than pristine PCBM.

Fig. 5 shows $J V$-curves, measured under AM1.5 illumination, of ITO/PEDOT:PSS/TQ1:PCBM/LiF/Al solar cells whose active layer has been exposed to light in air for different times prior to the cathode deposition. The curves for the best performing devices are shown in Fig. 5. The photovoltaic parameters (mean values \pm standard deviations, each for 16 devices) are presented in Table 1. All photovoltaic parameters decrease by the exposure, but it is the photocurrent that is most severely affected. The $J_{\mathrm{SC}}$ is reduced by a factor of 2 after 2 hours of exposure while the $V_{\mathrm{OC}}$ decreased by $17 \%$ and $\mathrm{FF}$ by $30 \%$. Analysis of the dark $J V$-curves (Fig. S1, ESI $\dagger$ ) also revealed that the series resistance in the device increases with exposure time. After 19 hours the solar cell efficiency is almost zero.

AFM images (ESI, $\dagger$ Fig. S2) show that the exposure to light in air has no discernible effect on the lateral morphology of the film surface.

\section{Discussion}

The changes to the NEXAFS spectrum of pristine TQ1 after exposure to AM1.5 light in air for different times (Fig. 2a) are negligible which tells us that the empty molecular states of the TQ1 molecule have a high photo-stability, also compared to other conjugated polymers. However, the photo-bleaching of the UV-Vis spectrum of TQ1 upon exposure to white light and air shows that the optical properties of TQ1 are far from stable. This is in agreement with Henriksson et al. who observed photo-bleaching of TQ1 and assigned it to chain scission due to photo-induced oxidation. ${ }^{13}$ According to Henriksson et al., the low energy absorption peak of TQ1 originates from the absorption of aromatic structures while the high energy absorption peak is due to intramolecular charge transfer along the backbone of the polymer. ${ }^{13}$ The exposure to light in air also results in a small shift of the low energy absorption peak towards higher energies. Density functional theory calculations by Hedström et al. have shown that the low energy absorption peak of TQ1 shifts to higher energies for shorter oligomers (oligomer sizes $n<10) .{ }^{39}$ Comparing the shift $(\sim 30 \mathrm{~nm})$ observed in our experiments with the calculated shifts, this would indicate that TQ1 is cut into quite short segments, which should likely affect also the electrical properties. The inclusion of $10 \mathrm{wt} \%$ PCBM into a TQ1 matrix enhances the stability towards photo-bleaching of the polymer (Fig. 3c). This is in agreement with the reports by Reese et al. for P3HT where the addition of PCBM also slows down the photo-bleaching of $\mathrm{P} 3 \mathrm{HT} .{ }^{17}$ We also observe changes to 
a)

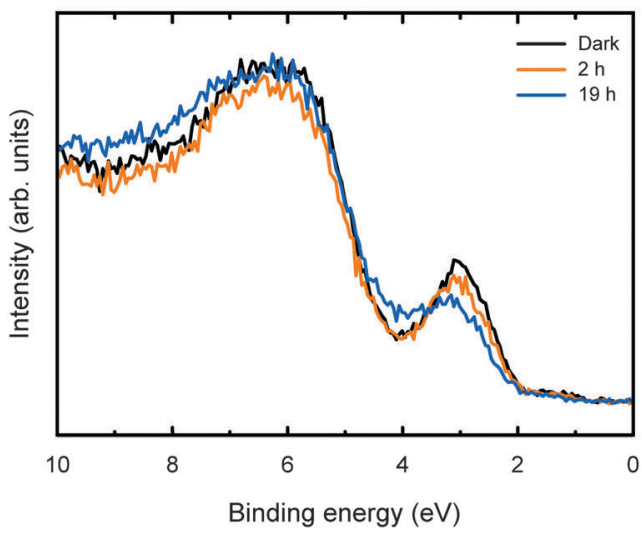

b)

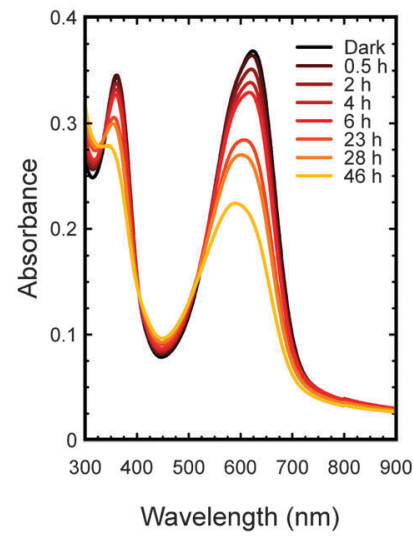

c)

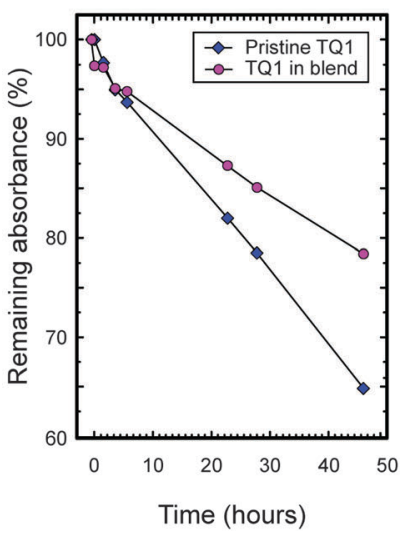

Fig. 3 Valence band (a) and UV-Vis absorption spectra (b) of pristine TQ1 films, unexposed to air and light (black), and after different exposure times to AM1.5 light in air (red to yellow). (c) Remaining absorbance, calculated from the area under the low energy absorption peak for pristine TQ1 (blue diamonds) and a TQ1: PCBM 9:1 w/w blend (purple circles) after different exposure times to AM1.5 light in air.

a)

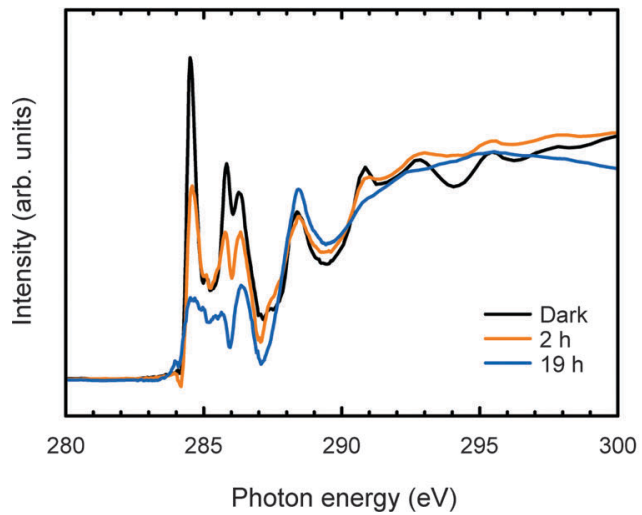

b)

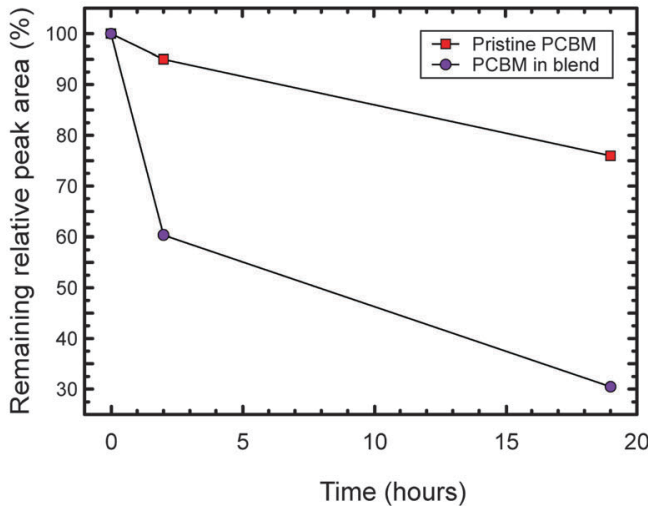

Fig. 4 (a) C1s NEXAFS spectral component originating from PCBM, extracted by subtraction of the TQ1 spectral contribution from experimental NEXAFS spectra of the TQ1: PCBM 1: 3 blend films (Fig. 2c) exposed to light and air for different times. (b) Remaining relative peak area of the $\pi^{\star}$ peak of PCBM at $284.5 \mathrm{eV}$ for pristine PCBM (red squares) and the PCBM component of a TQ1: PCBM 1:3 w/w blend (purple circles) after different exposure times to light in air.

the valence band spectrum of TQ1 upon light exposure in air, and note that the decrease in relative peak intensity of the HOMO ( $90 \%$ remaining intensity after $2 \mathrm{~h}$ and $75 \%$ after $19 \mathrm{~h}$ ) is similar in size to the decrease in relative peak intensity of the low energy absorption peak in the UV-Vis spectra (94\% remaining intensity after $2 \mathrm{~h}$ and $80 \%$ after $23 \mathrm{~h}$ ). Comparing the effect observed in the valence band and NEXAFS spectrum indicates that the lowest unoccupied molecular orbital (LUMO) of TQ1 is less susceptible to chemical alterations of the polymer molecules than the HOMO.

In contrast to TQ1, the changes to the NEXAFS spectrum of pristine PCBM after exposure to AM1.5 light in air for different times (Fig. 2b) are significant. Also, comparing the photo-induced changes in the valence band of TQ1 (Fig. 3a) to the substantial changes to the valence band spectrum of PCBM reported by Anselmo et al., ${ }^{24}$ clearly proves that the surface of PCBM degrades faster than the surface of TQ1.

How the NEXAFS spectrum of a TQ1:PCBM blend is affected by illumination in air is shown in Fig. 2c. Upon increased exposure, the PCBM-related resonances in the blend spectrum become weaker. This could have two possible causes: (1) exposure to light changes the vertical composition of the blend, as observed for P3HT:PCBM blends by Tournebize et al. ${ }^{19}$ or (2) PCBM in the blend degrades upon exposure, as it does in its pristine form. The first reason is less likely to occur for TQ1, because first, the glass transition temperature of P3HT is below room temperature, ${ }^{40}$ whereas that of TQ1 is $100{ }^{\circ} \mathrm{C},{ }^{41}$ hence substantially higher temperatures are needed to trigger any diffusion in a TQ1:PCBM blend than in a P3HT:PCBM blend. Secondly, if the decreased intensity of the PCBM-related resonances in the exposed blends compared to unexposed ones were a result of PCBM diffusing from the surface into the film, then the spectra shown in Fig. 4a would not look like that of pure PCBM. The same TQ1 component (namely that of the unexposed blend) is subtracted from each blend spectrum to give the spectra shown in Fig. 4a, so if PCBM had diffused from the surface into the film, more TQ1 would reside in the surface layer and there 


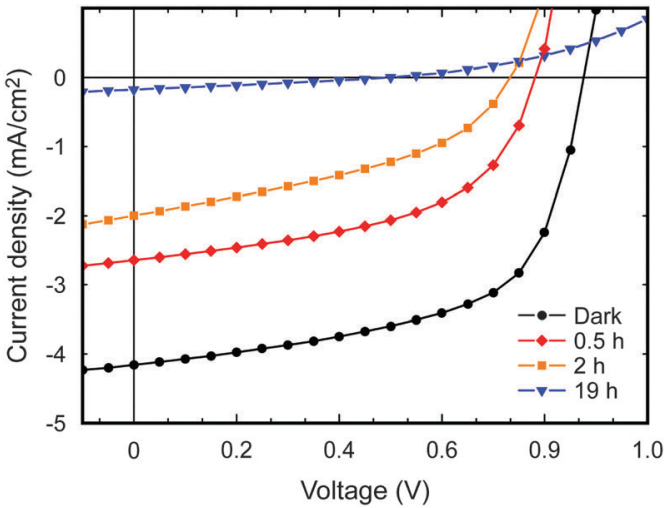

Fig. 5 Current-voltage characteristics measured under AM1.5 illumination of the best performing ITO/PEDOT:PSS/TQ1:PCBM/LiF/Al devices, unexposed to light and air, as well as exposed to light in air for different times prior to the cathode deposition.

would be a remaining TQ1 component visible in the spectra. Since this is clearly not the case, as can be seen by comparing Fig. 2 and 4a, we can confidently exclude alternative 1. Therefore we conclude that PCBM in the blend with TQ1 degrades upon exposure, just like PCBM does on its own.

To compare the degradation rates of pristine PCBM and PCBM in the blend, the PCBM component in the NEXAFS spectrum of the blend is extracted by subtracting the TQ1 component of the unexposed blend (obtained from the fit shown in Fig. S3 in the ESI $\dagger$ ) from the blend spectrum. Doing so, it is assumed here that TQ1 does not degrade in the blend. As stated earlier, this assumption is justified by two things: first, the NEXAFS spectrum of pristine TQ1 is unaffected by the exposure, and secondly, the UV-Vis experiments (Fig. $3 \mathrm{~b}$ and c) show that when TQ1 is in a blend with PCBM, the PCBM protects the TQ1 from degradation. It is worth noting, though, that when the TQ1 component is subtracted from the blend spectra, what remains is the PCBM component and the residual from the fit, but as can be seen (Fig. S3, ESI $\dagger$ ), the residual is minimal.

The resulting effect of illumination in air on the PCBM component in a TQ1:PCBM blend can be seen in the NEXAFS spectra in Fig. 4. Comparing this to the NEXAFS spectrum of pristine PCBM (Fig. 2b), it is clear that PCBM in a blend with TQ1 degrades faster (Fig. 4b) by light and air exposure than pristine PCBM. This is rationalized by the additional light absorption by TQ1 and subsequent electron transfer to the PCBM. Due to the extended absorption spectrum of TQ1 in the visible region, electrons are transferred to PCBM in the blend even by light with wavelengths in other regions of the solar spectrum (visible light up to $700 \mathrm{~nm}$ ) where pristine PCBM does not absorb. Reese et al. have previously attributed degradation of PCBM in a blend with P3HT to the photo-excitation of P3HT followed by photo-oxidation of PCBM. ${ }^{17}$ In a recent study by Yamane et al., the photo-oxidation of PCBM in blends with P3HT was investigated using matrix-assisted laser desorption/ ionization time-of-flight (MALDI-TOF) mass spectrometry, their findings suggesting that PCBM is stabilized by $\mathrm{P} 3 \mathrm{HT} .{ }^{23}$ What we concluded here from UV-Vis absorption and NEXAFS spectroscopy for TQ1 is the opposite to the conclusion of Yamane et al. On the other hand, TQ1 has been shown to have five times higher chemical stability than $\mathrm{P} 3 \mathrm{HT},{ }^{14}$ and looking at the NEXAFS and valence band spectra of pristine TQ1 and PCBM exposed to light in air, TQ1 is clearly more stable than PCBM, which suggests that, in the blend, it is PCBM rather than TQ1 that suffers worst from the exposure to light in air.

The effect of the active layers materials degradation on the solar cell device performance is illustrated by the $J V$-curves in Fig. 5. It is apparent that exposing the active layer to light in air before depositing the top electrode has a negative impact on the device performance. Considering how the empty states of the PCBM component in the surface layer of the blend were dramatically altered by the light exposure in air, it is not surprising that the device performance is also affected. The relative decrease in $J_{\mathrm{SC}}$ is significantly larger than the decrease in absorption due to the exposure to light in air of a TQ1 : PCBM $1: 3 \mathrm{w} / \mathrm{w}$ film (see Fig. S4, ESI $\dagger$ ). Other effects than the photobleaching must therefore also play an important role in the degradation of the solar cell performance. At the surface of the active layer, where the interface with the low work function electrode $(\mathrm{LiF} / \mathrm{Al})$ is made, electrons are extracted during the solar cell operation. Hence, if the electron-accepting material (PCBM) is destroyed at that interface, poor electron collection is to be expected, contributing to a lower $J_{\mathrm{SC}}$ and higher $R_{\mathrm{s}}$.

\section{Conclusions}

The photo-stability of the electronic structure of TQ1 in air surpasses that of PCBM. The LUMO of PCBM quickly changes upon exposure to light in air whereas that of TQ1 does not. PCBM blended with TQ1 photo-degrades at a higher rate than pristine PCBM, rationalized by the broader absorption spectrum of TQ1 and subsequent electron transfer to PCBM. Solar cells whose active layers have been exposed to light in air prior to the deposition of the top electrode show significantly reduced performance. Contributing factors to the decreased performance are the decreased absorption of TQ1 and the degradation of the

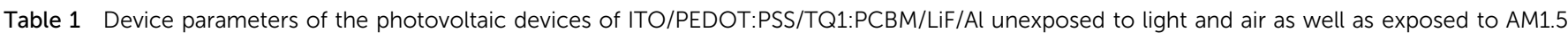
light in air for different times. Mean values \pm standard deviations, each from 16 devices are shown

\begin{tabular}{|c|c|c|c|c|c|}
\hline Exposure time & $J_{\mathrm{SC}}\left(\mathrm{mA} \mathrm{cm}{ }^{-2}\right)$ & $V_{\text {OC }}(\mathrm{V})$ & FF (\%) & $R_{\mathrm{s}}\left(\Omega \mathrm{cm}^{2}\right)$ & PCE (\%) \\
\hline Unexposed & $3.50 \pm 0.28$ & $0.88 \pm 0.02$ & $57.8 \pm 1.7$ & $2.8 \pm 0.6$ & $1.77 \pm 0.19$ \\
\hline $0.5 \mathrm{~h}$ & $2.39 \pm 0.14$ & $0.80 \pm 0.01$ & $48.7 \pm 2.9$ & $4.3 \pm 1.0$ & $0.93 \pm 0.09$ \\
\hline $2 \mathrm{~h}$ & $1.75 \pm 0.21$ & $0.73 \pm 0.02$ & $40.2 \pm 1.5$ & $7.0 \pm 1.0$ & $0.52 \pm 0.09$ \\
\hline $19 \mathrm{~h}$ & $0.13 \pm 0.03$ & $0.47 \pm 0.02$ & $27.7 \pm 0.7$ & $115 \pm 44$ & $0.017 \pm 0.004$ \\
\hline
\end{tabular}


electronic structure of PCBM in the blend film at the electron collecting interface.

\section{Acknowledgements}

The authors thank the beamline staff at D1011, MAX-IV Laboratory, for valuable technical advice with the NEXAFS experiments and Paul Beyer for assistance with the NEXAFS measurements. E. M. and E. W. acknowledge funding from the Swedish Energy Council and the Swedish Research Council. E. M. acknowledges also financial support from the Göran Gustafsson Foundation for Research in Natural Sciences and Medicine. A. O. acknowledges the Röntgen-Ångström-Cluster for financial support. The research benefits from valuable discussions within the framework of the COST Action MP1307 StableNextSol.

\section{References}

1 Y. Liu, J. Zhao, Z. Li, C. Mu, W. Ma, H. Hu, K. Jiang, H. Lin, H. Ade and H. Yan, Nat. Commun., 2014, 5, 1-8.

2 C. Liu, C. Yi, K. Wang, Y. Yang, R. S. Bhatta, M. Tsige, S. Xiao and X. Gong, ACS Appl. Mater. Interfaces, 2015, 7, 4928-4935.

3 F. C. Krebs, Stability and Degradation of Organic and Polymer Solar Cells, John Wiley \& Sons, 2012.

4 M. Jørgensen, K. Norrman, S. A. Gevorgyan, T. Tromholt, B. Andreasen and F. C. Krebs, Adv. Mater., 2012, 24, 580-612.

5 M. Jørgensen, K. Norrman and F. C. Krebs, Sol. Energy Mater. Sol. Cells, 2008, 92, 686-714.

6 M. P. de Jong, L. J. van Ijzendoorn and M. J. A. de Voigt, Appl. Phys. Lett., 2000, 77, 2255.

7 K. Kawano, R. Pacios, D. Poplavskyy, J. Nelson, D. D. C. Bradley and J. R. Durrant, Sol. Energy Mater. Sol. Cells, 2006, 90, 3520-3530.

8 K. Norrman and F. C. Krebs, Sol. Energy Mater. Sol. Cells, 2006, 90, 213-227.

9 A. Seemann, T. Sauermann, C. Lungenschmied, O. Armbruster, S. Bauer, H. J. Egelhaaf and J. Hauch, Sol. Energy, 2011, 85, 1238-1249.

10 S. Chambon, A. Rivaton, J.-L. Gardette, M. Firon and L. Lutsen, J. Polym. Sci., Part A: Polym. Chem., 2007, 45, 317-331.

11 M. Manceau, E. Bundgaard, J. E. Carlé, O. Hagemann, M. Helgesen, R. Søndergaard, M. Jørgensen and F. C. Krebs, J. Mater. Chem., 2011, 21, 4132-4141.

12 H. Hintz, H. J. Egelhaaf, H. Peisert and T. Chassé, Polym. Degrad. Stab., 2010, 95, 818-825.

13 P. Henriksson, C. Lindqvist, B. Abdisa, E. Wang, Z. George, R. Kroon, C. Müller, T. Yohannes, O. Inganäs and M. R. Andersson, Sol. Energy Mater. Sol. Cells, 2014, 130, 138-143.

14 J. E. Carlé, M. Jørgensen, M. Manceau, M. Helgesen, O. Hagemann, R. Søndergaard and F. C. Krebs, Sol. Energy Mater. Sol. Cells, 2011, 95, 3222-3226.

15 A. Rivaton, A. Tournebize, J. Gaume, P.-O. Bussière, J.-L. Gardette and S. Therias, Polym. Int., 2014, 63, 1335-1345.

16 N. Grossiord, J. M. Kroon, R. Andriessen and P. W. M. Blom, Org. Electron., 2012, 13, 432-456.
17 M. O. Reese, A. M. Nardes, B. L. Rupert, R. E. Larsen, D. C. Olson, M. T. Lloyd, S. E. Shaheen, D. S. Ginley, G. Rumbles and N. Kopidakis, Adv. Funct. Mater., 2010, 20, 3476-3483.

18 E. B. L. Pedersen, T. Tromholt, M. V. Madsen, A. P. L. Böttiger, M. Weigand, F. C. Krebs and J. W. Andreasen, J. Mater. Chem. C, 2014, 2, 5176-5182.

19 A. Tournebize, P.-O. Bussière, A. Rivaton, J.-L. Gardette, H. Medlej, R. C. Hiorns, C. Dagron-Lartigau, F. C. Krebs and K. Norrman, Chem. Mater., 2013, 25, 4522-4528.

20 E. Wang, L. Hou, Z. Wang, S. Hellstrom, F. Zhang, O. Inganas and M. R. Andersson, Adv. Mater., 2010, 22, 5240-5244.

21 Y. Kim, H. R. Yeom, J. Y. Kim and C. Yang, Energy Environ. Sci., 2013, 6, 1909-1916.

22 S. Chambon, A. Rivaton, J.-L. Gardette and M. Firon, Sol. Energy Mater. Sol. Cells, 2007, 91, 394-398.

23 S. Yamane, J. Mizukado, Y. Suzuki, M. Sakurai, L. Chen and H. Suda, Chem. Lett., 2015, 44, 339-341.

24 A. S. Anselmo, A. Dzwilewski, K. Svensson and E. Moons, PhD thesis, Karlstad University, 2013.

25 C. W. T. Bulle-Lieuwma, W. J. H. v. Gennip, J. K. J. v. Duren, P. Jonkheijm, R. A. J. Janssen and J. W. Niemantsverdriet, Appl. Surf. Sci., 2003, 203-204, 547-550.

26 J. W. Kiel, B. J. Kirby, C. F. Majkrzak, B. B. Maranville and M. E. Mackay, Soft Matter, 2010, 6, 641-646.

27 A. J. Clulow, A. Armin, K. H. Lee, A. K. Pandey, C. Tao, M. Velusamy, M. James, A. Nelson, P. L. Burn, I. R. Gentle and P. Meredith, Langmuir, 2014, 30, 1410-1415.

28 W. Kern and D. A. Puotinen, RCA Rev., 1970, 31, 187-206.

29 W. Kern, J. Electrochem. Soc., 1990, 137, 1887-1892.

30 G. Kissinger and W. Kissinger, Phys. Status Solidi A, 1991, 123, 185-192.

31 R. Hansson, L. K. E. Ericsson, N. P. Holmes, J. Rysz, A. Opitz, M. Campoy-Quiles, E. Wang, M. G. Barr, A. L. D. Kilcoyne, X. Zhou, P. Dastoor and E. Moons, J. Mater. Chem. A, 2015, 3, 6970-6979.

32 J. Stöhr, NEXAFS Spectroscopy, Springer-Verlag, Berlin, 1996.

33 B. Watts, L. Thomsen and P. C. Dastoor, J. Electron Spectrosc. Relat. Phenom., 2006, 151, 105-120.

34 B. Watts and H. Ade, J. Electron Spectrosc. Relat. Phenom., 2008, 162, 49-55.

35 D. S. Germack, C. K. Chan, B. H. Hamadani, L. J. Richter, D. A. Fischer, D. J. Gundlach and D. M. DeLongchamp, Appl. Phys. Lett., 2009, 94, 233303.

36 I. Brumboiu, A. S. Anselmo, B. Brena, A. Dzwilewski, K. Svensson and E. Moons, Chem. Phys. Lett., 2013, 568-569, 130.

37 J. Díaz, S. Anders, X. Zhou, E. J. Moler, S. A. Kellar and Z. Hussain, Phys. Rev. B: Condens. Matter Mater. Phys., 2001, 64, 125204.

38 T. Tromholt, M. V. Madsen, J. E. Carlé, M. Helgesen and F. C. Krebs, J. Mater. Chem., 2012, 22, 7592.

39 S. Hedstrom, P. Henriksson, E. Wang, M. R. Andersson and P. Persson, Phys. Chem. Chem. Phys., 2014, 16, 24853-24865.

40 J. Zhao, A. Swinnen, G. Van Assche, J. Manca, D. Vanderzande and B. Van Mele, J. Phys. Chem. B, 2009, 113, 1587-1591.

41 R. Kroon, R. Gehlhaar, T. T. Steckler, P. Henriksson, C. Müller, J. Bergqvist, A. Hadipour, P. Heremans and M. R. Andersson, Sol. Energy Mater. Sol. Cells, 2012, 105, 280-286. 